\title{
A Case Study: State Estimation and Optimal Control of an Industrial Copper Electrowinning Process
}

\author{
M. Anushka S. Perera, Tor Anders Hauge, and Carlos Fernando Pfeiffer
}

\begin{abstract}
This paper discusses an industrial case study related to the topics mathematical modeling, state-parameter-disturbance estimation, optimal control of large-scale complex control systems and technical computing. The case study involves the copper electrowinning process, which is a part of the copper leaching plant at Glencore Nikkelverk, Kristiansand, Norway. Improved control of chemical compositions within the electrowinning process through an optimal control strategy is one of our objectives. We present a way to solve this particular control problem, and in principal, the same procedure can be adapted to handle any large-scale complex control problem. State-parameter-disturbance estimation is a sub-problem, and two state estimators --- a modified version of the Extended Kalman Filter (EKF) and the Moving Horizon Estimate (MHE) --- are used to reconstruct the system state using simulated input-output data. It is shown that the EKF fails to estimate one of the parameters unless the algorithm is modified by adding an instability term. The MHE offers promising results in estimating parameters as compared to the classical EKF. Furthermore, the MHE explicitly handle constraints, which is an advantage. We use Modelica (as a systematic and efficient modeling approach for large-scale systems), Python (as a free and powerful tool for technical computing), structural analysis and graph-theory as main ingredients in the development and this combination significantly ease the analysis and synthesis of large-scale control systems. In the essence, our aim is twofold: (1) to demonstrate a simple, but a useful procedure of automating large-scale complex (optimal) controller design and synthesis and parameter estimation using available analytical and free computer-aided tools; and (2) to highlight the need of developing interfaces between Modelica/Simscape or similar modeling standards with a powerful programming language for technical computing, for example Python/MATLAB.
\end{abstract}

Index Terms-Controllability and observability, large-scale control systems, state and parameter estimation, technical computing.

\section{INTRODUCTION}

We present a solution to an Optimal Control Problem (OCP) [1] related to a real chemical process which is a part of the copper leaching plant at Glencore Nikkelverk, Kristiansand, Norway. The paper has two main considerations: (1) deploying available analytical tools in the analysis of large-scale complex control systems and (2) the software implementation.

It is beneficial to make use of a modeling language such as Modelica [4] or Simscape or similar for modeling of

Manuscript received May 1, 2016; revised July 12, 2016.

M. Anushka S. Perera, Carlos Fernando Pfeiffer are with the University College of Southeast Norway (e-mail: anushka_mrt@yahoo.com, anushka.perera@hit.no,carlos.pfeiffer@hit.no).

Tor Anders Hauge is with Glencore Nikkelverk, Kristiansand, Norway (e-mail: tor.hauge@glencore.no). large-scale complex physical systems. The Simscape language is based on the MATLAB which is a commercial software. We consider only non-commercial tools in this paper. Modelica is free and is an object-oriented, declarative, multi-domain modeling language for component oriented modeling. These features makes Modelica a systematic and efficient modeling tool. A system model encoded in Modelica is translated via Modelica based simulation tools such as Dymola, OpenModelica and JModelica.org (or CasADi [5]) to a set of differential, algebraic and discrete equations (so-called flat hybrid DAEs). If possible, it is useful to make flat hybrid DAEs available for general use without merely limiting to model simulations, optimizations, etc. One such attempt is CasADi where Modelica models can be imported to Python as flat hybrid DAEs. We emphasis the importance of developing interfaces between Modelica (or similar) and software tools for technical computing such as Python, MATLAB (has its own symbolic core and provides the Simscape language which supports object-oriented, declarative, multi-domain and component based modeling), Maple (inherently a symbolic framework) or similar.

Scale and complexity of nonlinear control systems determines the viability of implementing any theoretical analysis considering the entire system as a whole. Hence, as a first step it is often necessary to exploit the system structure [6] of a given large-scale complex control system prior to implementing any algebraic analysis such as algebraic observability analysis. Structurally decomposing a given large-scale complex system into subsystems, and then using those subsystems in the analysis and synthesis is one possibility. We demonstrate the usage of structural analysis by considering an electrowinning process.

Improved control of chemical compositions within the electrowinning process through an optimal control strategy is the control objective. Classical PID controllers may perform poorly on nonlinear Multiple-Input and Multiple-Output (MIMO) systems and they do not explicitly handle input, output and state constraints. Also, controller tuning in the presence of many PID controllers can be tricky. In order to avoid such drawbacks in classical controllers, an optimal control [7], [8] strategy, such as Nonlinear Model Predictive Control (NMPC) [9], can be considered. An OCP consists of two main subproblems: (1) observability analysis with respect to state-parameter-disturbance estimation [10], and (2) controllability analysis. These subproblems are dealt with in detail by exploiting the structural properties of the model. We consider two state estimators: the Extended Kalman Filter (EKF) [10]-[13] and the Moving Horizon Estimate [14]. In our case study, it is found that the EKF is unable to estimate one of the parameters whilst the Moving Horizon Estimate provides accurate estimates for all state variables, parameters 
and disturbance variables. Inclusion of an instability [15] term to the EKF completely solve the divergence problem. More details about the divergence properties of the EKF can be found in [16][17]. The structure of the paper is as follows:

- Section II presents a mechanistic model for the copper electrowinning process.

- Section III discusses state estimation and optimal control.

- Section IV explains a software implementation.

- Section V shows the results.

- Section VI is for conclusion and future work.

\section{MATHEMATICAL MODEL}

A mechanistic model for the copper leaching process is available in [2]. See Fig. 1 for the process flow sheet. The model is given by Eqs. 1 to 12. There are four measurements: $y_{0}, y_{1}, y_{2}$ and $y_{3}$ (see Eq. 20). In order to control the chemical compositions in the electrowinning sections, sulfuric acid (sulfuric acid flow rate is $\dot{V}_{a}$ ) and powdered raw material which contains copper oxide (mass flow rate is $\dot{m}_{c}$ ) are added in the slurrification and leaching sections respectively. There are time delays - according to process experience time delays vary around 2 hours - before $\dot{V}_{a}$ and $\dot{m}_{c}$ affect the chemical compositions in the third buffer tank $V_{p b}^{(3)}$. In this paper, $\mathrm{H}_{2} \mathrm{SO}_{4}$ and $\mathrm{CuSO}_{4}$ compositions in the third buffer tank are taken as two control variables $u_{2}$ and $u_{3}$ (i.e. delays are not considered.) Tank volumes (or levels) are controlled by $u_{0}$ and $u_{1}$ (see Eq. 16). $w^{(1)}$ is the unknown disturbance vector (see Eq. 17) and $w^{(2)}$ is the known disturbance vector (see Eq. 18). The volume of the electrowinning tank $V_{e w}$ and $\eta$ are unknown parameters and these parameters are augmented as state variables - see Eqs. 11 and 12. $p$ is the parameter vector given in Eq. 19 .

$$
\begin{aligned}
& \frac{d}{d t} V_{e d}=\dot{V}_{p 2 e}+\dot{V}_{e m 2 d}-\dot{V}_{e d 2 m}-\dot{V}_{e d 2 w} \\
& \frac{d}{d t} \rho_{e d, \mathrm{CuSO} 4}=\frac{\dot{V}_{p 2 e} \cdot\left(\rho_{p b, \mathrm{CuSO}}^{(3)}-\rho_{e d, \mathrm{CuSO}}\right)}{V_{e d}} \\
& +\frac{\dot{V}_{e m 2 d} \cdot\left(\rho_{e m, C u S O 4}-\rho_{e d, C u S O 4}\right)}{V_{e d}} \\
& \frac{d}{d t} \rho_{e d, H 2 S O 4}=\frac{\dot{V}_{p 2 e} \cdot\left(\rho_{p b, H 2 S O 4}^{(3)}-\rho_{e d, H 2 S O 4}\right)}{V_{e d}} \\
& +\frac{\dot{V}_{e m 2 d} \cdot\left(\rho_{e m, H 2 S O 4}-\rho_{e d, H 2 S O 4}\right)}{V_{e d}} \\
& \frac{d}{d t} \rho_{e w, C u S O 4}=\frac{\dot{V}_{e d 2 w} \cdot\left(\rho_{e d, C u S O 4}-\rho_{e w}, \mathrm{CuSO4}\right)}{V_{e w}} \\
& +\frac{\dot{V}_{\text {vap }} \cdot \rho_{\text {ew }, \text { CuSO4 }}-\left(\frac{M_{C u S O 4}}{10^{3}}\right) \cdot\left(\frac{\dot{m}_{C u, o}}{M_{C u}}\right)}{V_{e w}} \\
& \frac{d}{d t} \rho_{e w, H 2 S O 4}=\frac{\dot{V}_{e d 2 w} \cdot\left(\rho_{e d, H 2 S O 4}-\rho_{e w, H 2 S O 4}\right)}{V_{e w}} \\
& +\frac{\dot{V}_{\text {vap }} \cdot \rho_{e w, H 2 S O 4}+\left(\frac{M_{H 2 S O 4}}{10^{3}}\right) \cdot\left(\frac{\dot{m}_{C u, o}}{M_{C u}}\right)}{V_{e w}} \\
& \dot{V}_{e d 2 w}=\dot{V}_{e w 2 m}+\dot{V}_{\text {vap }}
\end{aligned}
$$

$$
\begin{gathered}
\frac{\dot{m}_{C u, o}}{M_{C u}}=\underbrace{\frac{3600 \cdot \bar{\eta}}{z_{C u} \cdot C} \cdot \bar{I}}_{\eta} \\
\frac{d}{d t} V_{e m}=\dot{V}_{e d 2 m}+\dot{V}_{e w 2 m}+\dot{V}_{w 2 e m}-\left(\dot{V}_{e 2 s}+\dot{V}_{e m 2 d}+\dot{V}_{e m 2 b l}\right) \\
\frac{d}{d t} \rho_{e m, C u S O 4}=\frac{\dot{V}_{e d 2 m} \cdot\left(\rho_{e d, C u S O 4}-\rho_{e m, C u S O 4}\right)}{V_{e m}} \\
+\frac{\dot{V}_{e w 2 m} \cdot\left(\rho_{e w, C u S O 4}-\rho_{e m, C u S O 4}\right)}{V_{e m}} \\
\frac{\dot{V}_{w 2 e m} \cdot \rho_{e m, C u S O 4}}{V_{e m}} \\
+\frac{\dot{V}_{e d 2 m} \cdot\left(\rho_{e d, H 2 S O 4}-\rho_{e m, H 2 S O 4}\right)}{V_{e m}} \\
-\frac{\dot{V}_{e w 2 m} \cdot\left(\rho_{e w, H 2 S O 4}-\rho_{e m, H 2 S O 4}\right)}{V_{e m}} \\
\frac{\dot{V}_{w 2 e m} \cdot \rho_{e m, H 2 S O 4}}{V_{e m}} \\
\frac{d}{d t} \eta=0 \\
\frac{d}{d t} V_{e w}=0
\end{gathered}
$$

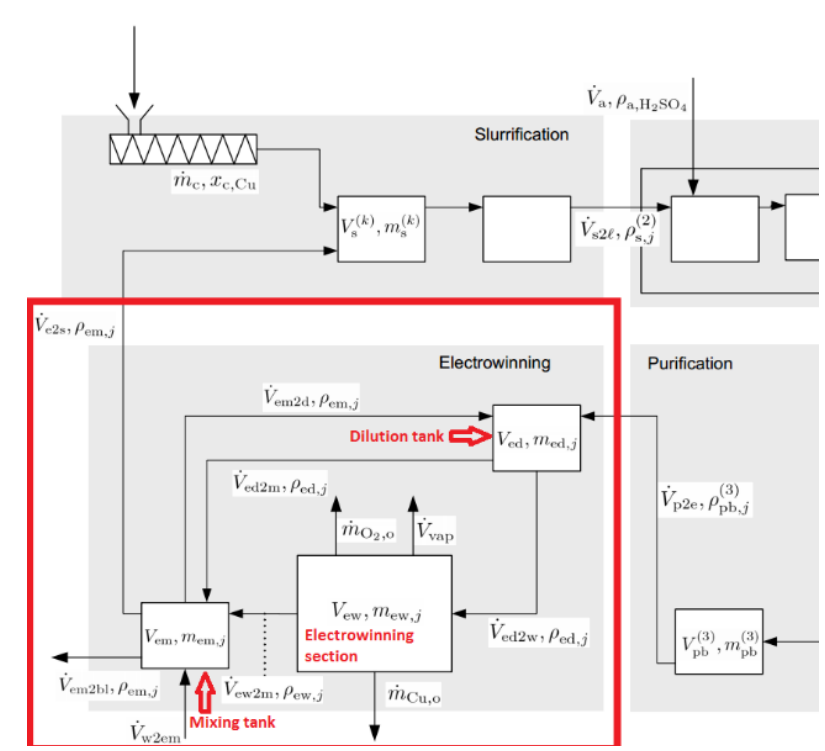

Fig. 1. A part of the flow sheet for the copper leaching process (taken from [16])

Corresponding noisy system is given in Eq. 21, where $v$ is the measurement noise. The state vector $x$ is given in Eq. 15 . $f$ and $h$ are given functions while $\psi$ is usually not known Unknown disturbance may be modeled as

$$
\dot{w}^{(2)}=\psi\left(w^{(2)}, \beta\right)+\varepsilon
$$

where $\beta$ is given parameter vector and $\varepsilon$ is a given white noise process (see Eqs. 13 and14). See [18] for one possible disturbance model. ${ }^{1}$ Eqs. 13 and 14 give possible disturbance models for $\dot{V}_{e d 2 w}$ and $\dot{V}_{e w 2 m}$. Eq. 22 gives the discrete version of Eq. 21 - where, $x \equiv x(t), x_{k} \equiv x\left(t_{k}\right)$ and $t_{k}=k . \Delta t$ for $k \in \mathbb{Z}_{\geq 0} . t$ is discretized with the time step $\Delta t$. $f_{k}, \psi_{k}$ and $h_{k}$ are some functions. $u(t)$ and $w^{(1)}(t)$ are

\footnotetext{
${ }^{1}$ A notation: $n_{a}$ gives the number of elements of the vector $a$.
} 
taken to be piecewise constant functions - i.e., $u(t)=u_{k}$ and $w^{(1)}(t)=w_{k}^{(1)}$ are constants for $t \in[k \cdot \Delta t,(k+1) . \Delta t]$.

$$
\begin{aligned}
& \frac{d}{d t} \dot{V}_{e d 2 w}=-\underbrace{\beta_{\dot{V}_{e d 2 w}}}_{\geq 0} \dot{V}_{e d 2 w}+\varepsilon_{V_{e d 2 w}} \\
& \frac{d}{d t} \dot{V}_{e w 2 m}=-\underbrace{-\beta_{\dot{V}_{e w 2 m}}}_{\geq 0} \dot{V}_{e w 2 m}+\varepsilon_{\dot{V}_{e w 2 m}} \\
& \left.x_{\text {max }} \geq x=\mid \begin{array}{c}
V_{e d} \\
V_{e m} \\
\rho_{e d}, \text { CuSO4 } \\
\rho_{\text {ew }, \text { CuSO4 }} \\
\rho_{\text {em }, \text { CuSO4 } 4} \\
\rho_{\text {ed }, \mathrm{H} 2 \mathrm{SO} 4} \\
\rho_{\text {ew }, \mathrm{H} 2 \mathrm{SO} 4} \\
\rho_{\text {em }, \mathrm{H} 2 \mathrm{SO} 4}
\end{array}\right\rfloor \geq x_{\text {min }}>0 \\
& u_{\text {max }} \geq u=\left[\begin{array}{c}
\dot{V}_{p 2 e} \\
\dot{V}_{e 2 s} \\
\rho_{p b, \mathrm{CuSO}}^{(3)} \\
\rho_{p b, \mathrm{H} 2 \mathrm{SO} 4}^{(3)}
\end{array}\right] \geq u_{\text {min }}>0 \\
& w^{(1)}=\left[\begin{array}{c}
\dot{V}_{e m 2 d} \\
\dot{V}_{e d 2 m} \\
I \\
\dot{V}_{w 2 e m} \\
\dot{V}_{e m 2 b l}
\end{array}\right]>0 \\
& w^{(2)}=\left[\begin{array}{c}
\dot{V}_{e d 2 w} \\
\dot{V}_{e w 2 m}
\end{array}\right]>0 \\
& p=\left\lfloor\begin{array}{c}
\eta \\
V_{e w}
\end{array}\right\rfloor>0 \\
& y=\left|\begin{array}{c}
V_{e d} \\
V_{e m} \\
\frac{M_{C u}}{M_{C u S O 4}} \cdot \rho_{e w}, \mathrm{CuSO4} \\
\rho_{e w, H 2 S O 4}+\frac{M_{H 2 S O 4}}{M_{C u S O 4}} \cdot \rho_{e w, C u S O 4}
\end{array}\right|>0 \\
& \Sigma:\left\{\begin{array}{c}
\dot{x}=f\left(x, u, w^{(1)}, w^{(2)}, p\right) \\
\dot{p}=0 \\
\dot{w}^{(2)}=\psi\left(w^{(2)}, \beta\right)+\varepsilon \\
y=h(x)+v
\end{array}\right. \\
& \Sigma_{k}:\left\{\begin{array}{c}
x_{k+1}=f_{k}\left(\Delta t, x_{k}, u_{k}, w_{k}^{(1)}, w_{k}^{(2)}, p_{k}\right) ; k=0,1, \ldots \\
p_{k+1}=p_{k} ; k=0,1, \ldots \\
w_{k+1}^{(2)}=\psi_{k}\left(w_{k}^{(2)}, \beta\right)+\varepsilon_{k} ; k=0,1, \ldots \\
y_{k}=h_{k}\left(x_{k}\right)+v_{k} ; k=0,1, \ldots
\end{array}\right.
\end{aligned}
$$

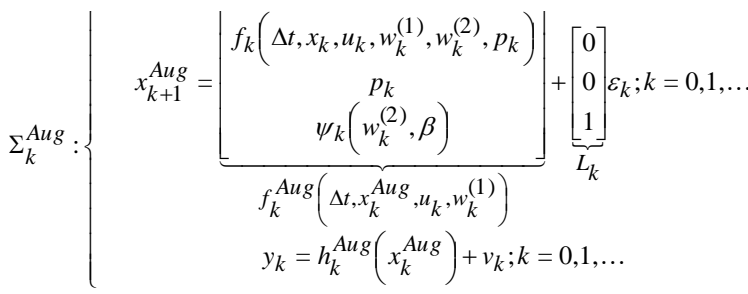

The elements of $x, u, w^{(1)}, w^{(2)}, w^{(1)}, p$, and $y$ are scaled with respect to their nominal values. For example,

$$
\rho_{e d, \mathrm{H}_{2} \mathrm{SO}_{4}}=\rho_{e d, \mathrm{H}_{2} \mathrm{SO}_{4}}^{0} \cdot \rho_{e d, \mathrm{H}_{2} \mathrm{SO}_{4}}^{S C}
$$

where $\rho_{e d, \mathrm{H}_{2} \mathrm{SO}_{4}}^{0}$ is the nominal value of $\rho_{e d, \mathrm{H}_{2} \mathrm{SO}_{4}}$ and such scaling makes $\rho_{e d, H_{2} S_{4}}^{S C}$ varies close to 1 . It is advisable to use scaling if the variables vary in vastly different scales.

\begin{tabular}{|c|c|l|}
\multicolumn{1}{c|}{ TABLE I: GIVES VARIABLES' UNITS } \\
\hline Variable & Units & \multicolumn{1}{c|}{ Description } \\
\hline$t$ & {$[h]$} & Time \\
\hline$V$ & {$\left[\mathrm{~m}^{3}\right]$} & Volume \\
\hline$\dot{V}$ & {$\left[\mathrm{~m}^{3} / \mathrm{h}\right]$} & Volumetric flow rate \\
\hline$\rho$ & {$[\mathrm{g} / \mathrm{l}]$} & Mass concentration \\
\hline$M$ & {$[\mathrm{~g} / \mathrm{mol}]$} & Molecular mass \\
\hline$Z$ & {$[-]$} & Number of valence electrons \\
\hline$I$ & {$[\mathrm{~A}]$} & Electric current \\
\hline $\bar{\eta}$ & {$[-]$} & Currency efficiency \\
\hline
\end{tabular}

\section{State Estimation AND Optimal CONTROL}

Consider the discrete stochastic system [13] in Eq. 22. The augmented state,

$$
x_{k}^{A u g}=\left[\begin{array}{c}
x_{k} \\
w_{k}^{(2)} \\
p_{k}
\end{array}\right]
$$

May be estimated from available measurements (if the augmented system is observable) $y_{i}$ for $i \leq k ; w_{i}^{(1)}$ for $i \leq k-1$; and $u_{i}$ for $i \leq k-1$. Various nonlinear state estimators are available, such as the Extended Kalman Filter (EKF), the Unscented Kalman Filter (UKF) [10], the Particle Filter (PF) [19][20][21], and the Moving Horizon Estimate (MHE). A combination of the MHE and the EKF (MHE-EKF combo) is used in this paper - EKF may be replaced by, for example, PF (i.e., MHE-PF combo). The success of a state estimation process demands three things: (1) state observability, (2) state stochastic observability, (3) and stochastic controllability. Stochastic controllability associates with the excitation of state variables by process noises variables - more precisely, unstable state variables should be controllable with respect to process noise variables to achieve stochastic controllability. Note that some choices of $\psi$ may cause the augmented system unobservable, hence it should be defined carefully. The augmented noisy system which is used in state-parameter-disturbance estimation is given in Eq. 23. ${ }^{2}$

Fig. 2 gives an EKF implementation. $z_{k}^{A u g+}$ is an estimate for $x_{k}^{A u g} . A_{k-1}$ matrix is adjusted by an instability term $\alpha I$ $(\alpha \geq 0)$ and this improves the stability of EKF [22][25]. If $\alpha=0$, then we have the conventional EKF. It is seen that $\alpha \neq 0$ is advisable especially for parameter estimation.

${ }^{2} 0$ and 1 are used both as scalars and (zero and unit) matrices where necessary and reader is advised to pick the correct one. 
Notice that $\varepsilon$ excites the system state $x$ via $w^{(2)}$. Even though the unstable modes of the augmented system is controllable with respect to $\varepsilon$, it is still appropriate to add additional fictitious noise [10] variables to every state equation in the augmented system. Fictitious noise variables improve the stability of EKF - i.e., Eq. 21 becomes $\dot{x}=f\left(x, u, w^{(1)}, w^{(2)}, p\right)+\varepsilon_{x}$ and $\dot{p}=\varepsilon_{p}$, where $\varepsilon_{x}$ and $\varepsilon_{p}$ are fictitious noise vectors.

$$
\begin{aligned}
& A_{k-1}=\left.\frac{\partial}{\partial x_{k-1}^{A u g}} f_{k-1}^{A u g}\left(\Delta t, x_{k-1}^{A u g}, u_{k-1}, w_{k-1}^{(1)}\right)\right|_{z_{k-1}^{A u g}+} \\
& A_{k-1}^{\alpha}=A_{k-1}+\alpha I ; \alpha \geq 0 \\
& Q_{k-1} \geq 0 \text { is given. } \\
& P_{k}^{-}=A_{k-1}^{\alpha} P_{k-1}^{+} A_{k-1}^{\alpha T}+L_{k-1} Q_{k-1} L_{k-1}^{T} \\
& P_{k}^{-}=\left(P_{k}^{-}+P_{k}^{-T}\right) / 2 \\
& z_{k}^{A u g-}=f_{k-1}^{A u g}\left(\Delta t, z_{k-1}^{A u g+}, u_{k-1}, w_{k-1}^{(1)}\right) \\
& C_{k}=\frac{\partial}{\left.\partial x_{k}^{A u g} h\left(x_{k}^{A u g}\right)\right|_{z_{k}^{A u g-}} ^{A u g}} \\
& R_{k}>0 \text { is given. } \\
& K_{k}=P_{k}^{-} C_{k}^{T}\left(C_{k} P_{k}^{-} C_{k}^{T}+R_{k}\right)^{-1} \\
& z_{k}^{A u g}+=z_{k}^{\text {Aug }}+K_{k}\left[y_{k}-C_{k} z_{k}^{A u g-}\right] \\
& P_{k}^{+}=\left(I-K_{k} C_{k}\right) P_{k}^{-} \\
& P_{k}^{+}=\left(P_{k}^{+}+P_{k}^{+T}\right) / 2
\end{aligned}
$$

Fig. 2. An EKF implementation.

Consider $y(t)$ for $t_{k}-T \leq t \leq t_{k}$, where $T$ is a time horizon to the past $-T=N_{m h e} . \Delta t$ and $N_{m h e}$ is a positive integer. $x\left(t_{k}\right)$ and $w^{(2)}\left(t_{k}\right)$ are estimated such that $J_{m h e}$ (in Eq. 24) is minimized subjected to Eq. 23 and constraints given in Eqs. 15, 18 and 19. $\hat{z}_{k-N_{m h e}}$ should be known and it can be estimated using EKF or PF or other estimator. At the end of each sample time, the horizon is shifted one time step forward - i.e., $t \in\left[t_{k}+\Delta t-\mathrm{T}, t_{k}+\Delta t\right]-J_{m h e}$ is optimized again and continue. Weighting matrices $Q_{1}^{i}, Q_{2}^{i}$, and $Q_{3}^{i}$ are given. See Fig. 2 .

$$
\begin{aligned}
J_{m h e}= & \sum_{i=k-N_{m h e}}^{k-1} \varepsilon_{i}^{T} Q_{1}^{i} \varepsilon_{i}+ \\
& \sum_{i=k-N_{\text {mhe }}}^{k}\left(y_{i}-h_{i}^{A u g}\left(z_{i}\right)\right)^{T} Q_{2}^{i}\left(y_{i}-h_{i}^{A u g}\left(z_{i}\right)\right)+ \\
& \left(z_{i-N_{m h e}}-\hat{z}_{i-N_{m h e}}\right)^{T} Q_{3}^{i}\left(z_{i-N_{m h e}}-\hat{z}_{\left.i-N_{m h e}\right)}\right.
\end{aligned}
$$

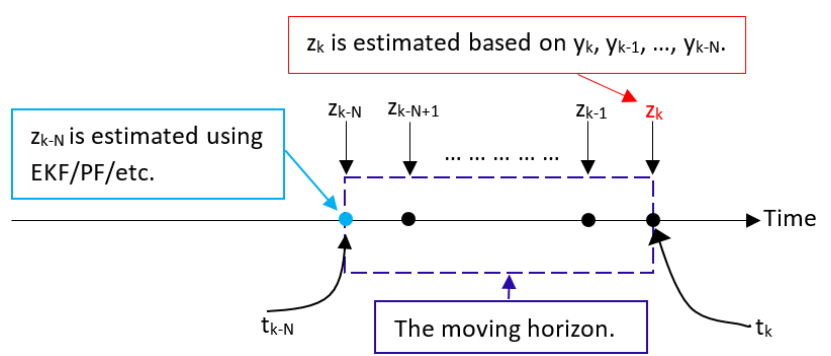

Fig. 3. MHE implementation with EKF/PF/etc.
Assume the system given Eq. 21 is output controllable and there exists an equilibrium point such that $\dot{x}=$ $f\left(x^{0}, u^{0}, w^{(1), 0}, w^{(2), 0}, p^{0}\right)=0$ and $y^{0}=h\left(x^{0}\right)+0$, $\forall t \geq 0$. Eq. 25 gives the linear model in deviation form. One possibility is to use the linear model to design an optimal controller.

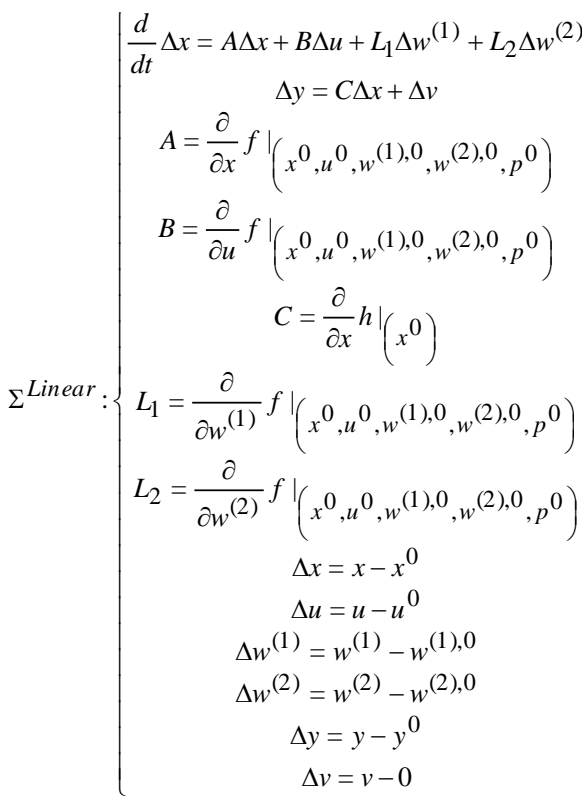

$$
\begin{aligned}
& \frac{d}{d t} z_{i}=\Delta r_{i}-\Delta y_{i} ; k=0,1, \ldots, n_{y} \\
& \Delta r_{i}=r_{i}-r_{i}^{0} \\
& \Delta y_{i}=y_{i}^{f i l t}-y_{i}^{0} \\
& z=\left[\begin{array}{c}
z_{0} \\
z_{1} \\
\cdots \\
z_{n}
\end{array}\right]
\end{aligned}
$$

It is necessary to include integral actions in order to achieve an offset-free reference tracking (the Magic of Integral Control [23]) and better disturbance rejection. See Eq. 26 , where $r_{i}$ is the reference of $y_{i}$ and $y_{i}^{\text {filt }}$ is the (lowpass) filtered signal of $y_{i}$. Refer [24] and [25] for more details. $\Delta w^{(2)}$ is augmented appropriately - see Eq. 28. $\Delta w^{(2)}$ is not controllable by $\Delta u$, hence a model for $\Delta w^{(2)}$ is defined such that $\Delta w^{(2)}$ is stable. Also some rate control is included (see Eq. 27).

$$
\frac{d}{d t} \Delta u=\tilde{u}
$$

$$
\frac{d}{d t} \Delta w^{(2)}=\left[\begin{array}{cc}
-\underbrace{\beta_{0}}_{0<\beta_{0}} & 0 \\
0 & -\underbrace{\beta_{0}}_{0<\beta_{1}<1}
\end{array}\right] \Delta w^{(2)}
$$

Now, the cost function given in Eq. 29 is minimized subjected to Eqs. 25, 26, 27 and 28. $Q_{\Delta w^{(2)}}$ could be set to zero since it may not be necessary to optimize $\Delta w^{(2)}$.

$$
J_{\infty}={ }_{t_{f} \rightarrow \infty}^{\operatorname{limit}} \int_{0}^{t} f(t) d t
$$




$$
\begin{aligned}
& J(t)=\tilde{x} Q_{\tilde{x}} \tilde{x}^{T}+\tilde{u} Q_{\tilde{u}} \tilde{u}^{T} \\
& \left.\tilde{x}=\mid \begin{array}{c}
\Delta x \\
\Delta w^{(2)} \\
z \\
\Delta u
\end{array}\right\rfloor \\
& Q_{\tilde{x}}=\left|\begin{array}{cccc}
Q_{\Delta x} & 0 & 0 & 0 \\
0 & Q_{\Delta w}(2) & 0 & 0 \\
0 & 0 & Q_{z} & 0 \\
0 & 0 & 0 & Q_{\Delta u}
\end{array}\right| \\
& R A+A^{T} R-R B Q_{\tilde{u}}^{-1} B^{T} R+Q_{\tilde{x}}=0 \\
& K_{\infty}=Q_{\tilde{u}}^{-1} B^{T} R \\
& \tilde{u}=-K_{\infty} \tilde{x}=-\left[\begin{array}{llll}
K_{\infty}^{1} & K_{\infty}^{2} & K_{\infty}^{3} & K_{\infty}^{4} \\
\Delta \\
\Delta u \\
z u \\
w^{(2)} \\
z
\end{array}\right. \\
& =-K_{\infty}^{1} \Delta x-K_{\infty}^{2} \Delta w^{(2)}-K_{\infty}^{3} z-K_{\infty}^{4} \Delta u \\
& \Delta u=\int_{0}^{t} \tilde{u}(\tau) d \tau
\end{aligned}
$$

The analytical solution to unconstrained OCP is given by Eq. 30. On the other hand, it is possible to consider a finite horizon OCP - i.e.,

$$
J_{t_{f}}=\int_{0}^{t} f^{<\infty} J(t) d t
$$

Unconstrained infinite horizon problem is much faster to implement. $Q_{\tilde{x}}$ and $Q_{\Delta u}$ are carefully tuned. $\Delta x$ and $\Delta w^{(2)}$ are replaced by their estimates. Alternatively, linear or nonlinear Model Predictive Controller (LMPC/NMPC) can be considered. Assume the current state $x_{k}$ is known (or estimated). Let, it be necessary to steer output from $y_{k}$ to $y_{k+M_{y}}\left(M_{y}<\infty\right.$ is the prediction horizon and it is a positive integer) in finite time by manipulating a sequence of control variables $\left\{u_{k}, u_{k+1}, \cdots, u_{k+M_{u}-1}\right\}$, where $M_{u}$ is control horizon $\left(M_{u} \leq M_{y}\right) . \Delta u_{i}=u_{i}-u_{i-1}$ for $i=k, k+$ $1, \cdots, k+M_{u}-1, \Delta u_{i}=0$ for $i \geq k+M_{u}$ and $u_{-1}$ is given There could be many possibilities of choosing a control sequence to achieve the same output transition. In order to pick one of the input trajectories, additional constraints can be imposed on input variables. This is the basic idea in optimal control, where a cost function is defined and minimized with respect to $u(t)$ for $t \in\left[t_{k}, t_{k+M_{y}}\right]$. The cost function can be defined in many flavors. Eq. 31 gives an example.

$w^{(1)}$ and $w^{(2)}$ affect the performance of the controller. One way to tackle the problem is to assume $w^{(2)}$ is a random process and $w^{(1)}(t)=w_{k}^{(1)}$ for $t \in\left[t_{k}, t_{k}+M_{y} . \Delta t\right]$. This makes $J_{n m p c}$ random. We may, among other options, optimize the expected value of the cost function - i.e., $E\left\{J_{n m p c}\right\}$. Stochastic optimal control strategies are discussed in [7]. Instead, we consider some estimate of $w_{k}^{(2)}$ - say $\widehat{w}_{k}^{(2)}-$ and $w^{(2)}(t)=w_{k}^{(2)}$ for $t \in\left[t_{k}, t_{k}+M_{y} . \Delta t\right]$, in order to obtain a deterministic cost function. Once the OCP is solved (for given $x_{k}$ or an estimate of it $x_{k}$ ), the optimal control sequence $\left\{u_{k}^{*}, u_{k+1}^{*}, \cdots, u_{k+M_{u}-1}^{*}\right\}$ as well as $\left\{x_{k+1}^{*}, x_{k+2}^{*}, \cdots, x_{k+M_{y}}^{*}\right\}$ are found. We set the current control action to be $u_{k}=u_{k}^{*}$ for $t \in\left[t_{k}, t_{k}+\Delta t\right]$. An estimate of $p_{k}$ used in the OCP implementation as well.

$$
\begin{aligned}
& J_{n m p c}=\sum_{k=0}^{k=M_{y}-1}\left(y_{k+1}-y_{k+1}^{r e f}\right)^{T} W^{y}\left(y_{k+1}-y_{k+1}^{r e f}\right) \\
&+\sum_{k=0}^{k=M_{u}-1} \Delta u_{k}^{T} W^{u} \Delta u_{k} \\
& x_{k+i}- \\
& f_{k+i-1}\left(\Delta t, x_{k+i-1}, \Delta u_{k+i-1}+u_{k+i-2}, w_{k}^{(1)}, \hat{w}_{k}^{(2)}, \hat{p}_{k}\right)=0 ; i=1,2, \ldots, M_{y} \\
& u_{k+i}=\Delta u_{k+i}+u_{k+i-1} ; i=0,1, \ldots, M_{y}-1 \\
& \Delta u_{k+i}=0 ; i=M_{u}, M_{u}+1, \ldots, M_{y}-1 \\
& x_{k+i} \geq 0 ; i=1,2, \ldots, M_{y} \\
& u_{k+i}^{\min } \leq u_{k+i} \leq u_{k+i}^{\max } ; i=0,1, \ldots, M_{y}-1
\end{aligned}
$$

Analysis and synthesis of OCPs involves controllability and observability analysis. In this paper, we will not discuss algebraic observability and controllability [26], [27], instead we exploit the structural observability and controllability [28][29][6]. Observability of the noise-free system must be fulfilled first. Consider the noise-free form of Eq. 21 (by setting $\varepsilon=v=0$ ) given in Eq. 35 .

$$
\Sigma_{n f}:\left\{\begin{array}{c}
\dot{x}_{n f}=f\left(x_{n f}, u, w^{(1)}, w_{n f}^{(2)}, p_{n f}\right) \\
\dot{p}_{n f}=0 \\
\dot{w}_{n f}^{(2)}=\psi\left(w_{n f}^{(2)}, \beta\right) \\
y_{n f}=h\left(x_{n f}\right)
\end{array}\right.
$$

The augmented state $x_{n f}^{A u g}(t)$ moves on its state space, starting at $x_{n f}^{A u g}(t=0)$. The trajectory of $x_{n f}^{A u g}(t)$ is shaped by $u(t)$ and $w^{(1)}(t)$. The output trajectory is given by $y_{n f}(t)=h\left(x_{n f}(t)\right)$. If (locally) no two distinct initial conditions give identical output trajectories for any admissible $u_{n f}(t)$ and $w_{n f}^{(1)}(t)$, then we say that the state are (locally) distinguishable. In other words:

$$
h\left(x_{n f}^{1}\right) \neq h\left(x_{n f}^{2}\right) \Rightarrow x_{n f}^{1} \neq x_{n f}^{2}, w_{n f}^{(2), 1} \neq w_{n f}^{(2), 2}, p_{n f}^{1} \neq p_{n f}^{2}
$$

State distinguishability property implies state observability. Note that $u_{n f}(t)$ and $w_{n f}^{(1)}(t)$ affect observability. If there exists an admissible control $u_{n f}(t)$ such that the state transition from any $x_{n f}^{1}$ to any state $x_{n f}^{2}$ is possible, then we say that the state is controllable $\left(w_{n f}^{(1)}(t)\right.$ and $w_{n f}^{(2)}(t)$ affect controllability). The reader is refereed to [27][30] for a detailed discussion on nonlinear observability and controllability. Often, implementing algebraic tests for observability and controllability analysis on large-scale complex systems are tedious or even practically impossible. A practical solution is first to analyze large-scale systems for structural observability and controllability, followed by algebraic tests for structurally decomposed subsystems as necessary. Structural observability (controllability) gives a necessary condition for observability (controllability). 
Interestingly, there is a graph-theoretic analogy for structural analysis [6]. ${ }^{3}$

Structural observable analysis involves structural dependencies among state and measurement variables and those dependencies are encoded into a directed graph $G_{O}$ (directed graph $=$ digraph). Nodes are the elements of the vectors $x_{n f}, w_{n f}^{(2)}, p_{n f}$ and $p_{n f}$. E.g., $x_{n f, i}$ is the i-th element of $x_{n f}$ and $x_{n f, i}$ is a node. Edges are defined in the following way:

- $\quad x_{n f, i} \rightarrow x_{n f, j}$ exists if $\partial f_{i} / \partial x_{n f, j} \neq 0$;

- $\quad x_{n f, i} \rightarrow w_{n f, j}^{(2)}$ exists if $\partial f_{i} / w_{n f, j}^{(2)} \neq 0$;

- $\quad x_{n f, i} \rightarrow p_{n f, j}$ exists if $\partial f_{i} / \partial p_{n f, j} \neq 0$;

- $\quad x_{n f, i} \rightarrow p_{n f, j}$ exists if $\partial f_{i} / \partial p_{n f, j} \neq 0$;

- $\quad w_{n f, i}^{(2)} \rightarrow w_{n f, j}^{(2)}$ exists if $\partial \psi_{i} / \partial w_{n f, j}^{(2)} \neq 0$;

- $y_{n f, i} \rightarrow x_{n f, j}$ exists if $\partial h_{i} / \partial x_{n f, j} \neq 0$.

Note that $a \rightarrow b$ denotes a directed edge from $a$ to $b$. Once the digraph is created, we can analyze for structural observability. It is a must that all state variables are reachable from outputs (output connectivity) - i.e. if there is at least one state variable such that there is no directed path from any of the output variables to that state's node, then the system is not structurally observable. However, this is a necessary condition for structural observability. A necessary and sufficient condition is that iff $G_{o}$ is spanned by cacti [28], then the system is structurally observable. A cactus contains a directed path, called a stem, starting from an output the root and ends at a state variable the top and cycles, called buds, which are attached to the stem and/or to other buds. See Fig. 4 for an example.

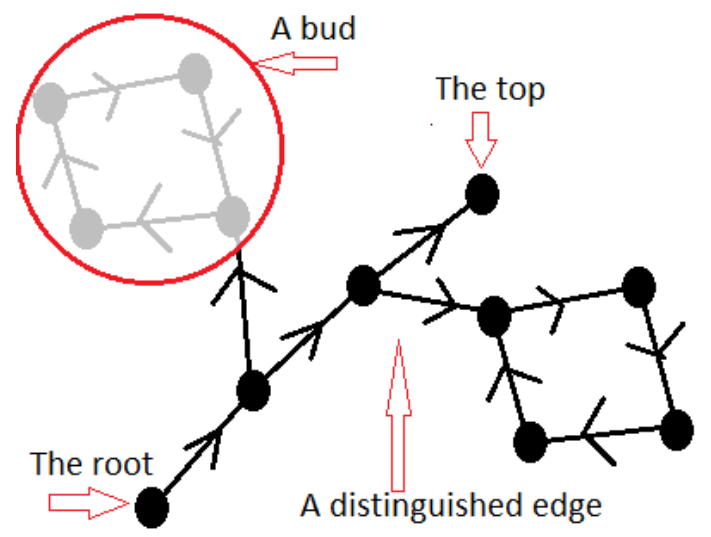

Fig. 4. A cactus with two buds.

Digraphs for structural controllability (with respect to control variables) analysis are created in a similar fashion. Nodes are the elements of the vectors $x_{n f}, u$ and $y_{n f}$. Let $G_{c}$ be the digraph for structural controllability analysis. The edges are defined in the following way:

$$
\begin{array}{ll} 
& x_{n f, j} \rightarrow x_{n f, i} \text { exists if } \partial f_{i} / \partial x_{n f, j} \neq 0 ; \\
\text { - } & u_{j} \rightarrow x_{n f, i} \text { exists if } \partial f_{i} / u_{j} \neq 0 ; \\
\text { - } & x_{n f, i} \rightarrow y_{n f, j} \text { exists if } \partial h_{j} / \partial x_{n f, i} \neq 0 .
\end{array}
$$

Iff $G_{C}$ is spanned by cacti, then the system is structurally output controllable. Similarly, state controllability can be defined. It is also possible to define structural controllability with respect to process noise $w_{n f}^{(2)}$ for stochastic controllability. Stochastic controllability affects convergence characteristic of the state estimation process [16], [32]. In some cases, structural observability (controllability) is sufficient for algebraic observability (controllability). For example, the system we consider in this paper is structurally observability and controllability and also structural properties provides sufficiency.

\section{SOFTWARE IMPLEMENTATION}

In this paper, usage of free software tools are emphasized. Python which is a powerful tool for technical computing is used as the scripting language. There are many Python packages which support, among others, various aspects systems and control engineering applications such as scipy, numpy, matplotlib, assimulo, casadi, pygraphviz, networkx, etc. For modeling of large-scale complex dynamic systems, Modelica specifications provide a better systematic approach. Several tools offer interfaces between Modelica and Python, for examples OpenModelica and JModelica.org. Modelica standards mainly focuses on modeling and simulation, however there exist an extension to Modelica, Optimica [33], which can handle OCPs. OpenModelica and JModelica.org support Optimica standards as well. CasADi - available as a Python package — is a symbolic framework for numerical optimization and it is conveniently possible to cast a general OCP using CasADi. Also, it is always possible to solve a general OCP via SciPy.

First, a Modelica ${ }^{4}$ package is created which contains the copper leaching process model EW and EW_Init (the steady state model) which extends EW. ${ }^{5}$ The key point here is that we can encode large-scale complex systems systematically using Modelica standards - in other words exploiting the modeling power in Modelica. To calculate a steady state, EW_Init is compiled using JModelica.org — the pymodelica package is used for compiling — and the compiled model is imported back, as a JMUModel object and called it init_model, to Python through pyjmi. Calling the method initialize() initializes EW_Init and thereby, a steady state is found. The method initialize() uses IPOPT solver [34]. There are many other alternatives which can be used in steady state calculation for instances CasADi and SciPy. EW is also used to generate necessary digraphs for structural observability and controllability analysis. This is done via CasADi by importing Modelica model into Python as symbolic DAEs, processing them (e.g. index reduction, etc.), generating and analyzing digraphs using networkx package.

The module scipy.optimize is used in the implementations of optimal control and moving horizon estimate. For example, scipy.optimize.fmin_cobyla() is a nonlinear optimizer which can be used for constrained optimization. An alternatively is IPOPT optimizer (through CasADi). IPOPT-CasADi combination needs lesser computational time as compared to fmin_cobyla()-Python. The EKF is straight forward to automate.

\footnotetext{
${ }^{3}$ A way of automating structural analysis in Python, using Modelica-JModelica.org-CasADi, is given in [31].
} 


\section{RESUlts}

The volume of electrowinning tanks $V_{e w}$ determines the rate of change of the chemical compositions in electrowinning tanks. $V_{e w}$ is not precisely known. However, the experience shows that the time constant is around 2 hours, hence $V_{e w}$ is chosen accordingly. Note that variables are scaled with respect to an equilibrium point, thereby scaled variables have their nominal values equal to ones. The entire analysis is based on scaled variables.

Two of the eigenvalues of matrix $A$ are zeros and others are complex with real negative parts. Zero eigenvalues corresponds to $V_{e d}$ and $V_{e m}$. Due to the presence of zero eigenvalues, it is necessary to have shorter sampling time. $\Delta t=0.25[\mathrm{~min}]$ is selected.

\section{A. Structural Analysis}

Based on available measurements $\left(n_{y}=4\right)$ it is expected to estimate all state variables $\left(n_{x}=8\right)$ and additionally, if possible, disturbance variables $\left(n_{w^{(2)}}=2\right)$ and parameters $\left(n_{p}=2\right)$. Fig. 5 depicts the digraph for structural observability analysis. There exists a spanning cacti covering all vertices, hence the system is structurally observable. There could be more than one spanning cacti. Note that even though $\beta_{\dot{V}_{e w 2 m}}=\beta_{\dot{V}_{e d 2 w}}=0$ (in Eqs. 13 and 14), the augmented model maintains structural observability.

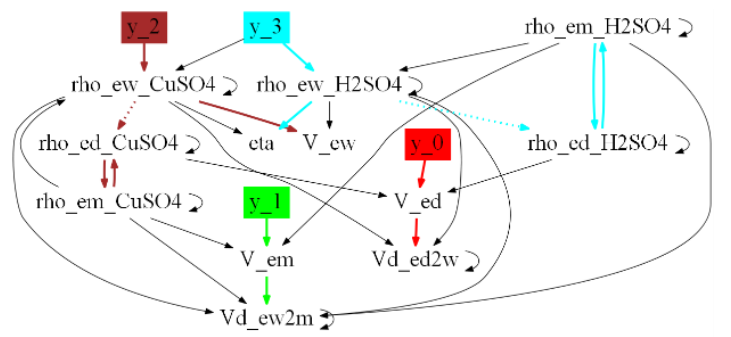

Fig. 5. Structural observability analysis: digraph is spanned by cacti covering all nodes.

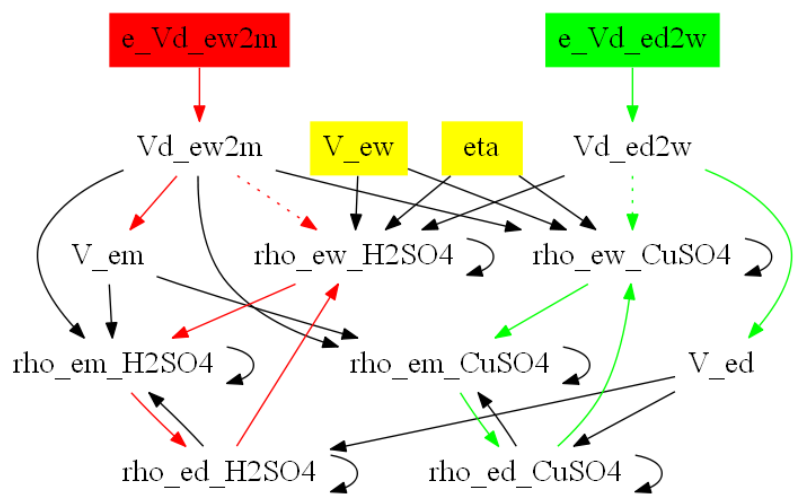

Fig. 6. Structural controllability analysis w.r.t. process noise variables. Nodes related to parameters are colored in yellow.

The digraph given in Fig. 6 is used to analyze for the controllability of state variables with respect to process noise variables. The objective is to check whether unstable modes are excited enough by process noise variables $\varepsilon_{\dot{V}_{e w 2 m}}$ and $\varepsilon_{\dot{V}_{e d 2 w}}$. It is seen that augmented state variables, except the state variables related to parameters, are controllable by process noise variables. If there is any unstable mode which is not controllable by process noise, then it is necessary to add some fictitious noise to it. It is always advisable to add fictitious noise processes to all state equations including Eqs.
11 and 12 (these equations links with model parameters). According to Fig. 7, it is clear that the system is structurally output controllable. In this case, the digraph can be divided into two sets $S_{1}$ and $S_{2}$ :

$$
\begin{aligned}
& S_{1}=\left\{y_{0}, y_{1}, V_{e d}, V_{e m}, u_{0}, u_{1}\right\} \\
& S_{2}=\left\{y_{2}, y_{3}, \rho_{e^{2}, \text { CuSO }_{4}}, \rho_{\text {ew }, \text { CuSO }_{4}}, \rho_{\text {em } \mathrm{CuSO}_{4}},\right. \\
& \left.\rho_{e d, H_{2} \mathrm{SO}_{4}}, \rho_{e w, \mathrm{H}_{2} \mathrm{SO}_{4}}, \rho_{e, \mathrm{H}_{2} \mathrm{SO}_{4}}, u_{2}, u_{3}\right\} \\
& \text { Vd_p2e } \\
& \text { rho_em_CuSO4 } 2 \text { y3 }
\end{aligned}
$$

Fig. 7. Structural (output) controllability analysis w.r.t. input variables.

There are no directed edges starting from $S_{2}$ and ending at $S_{1}$, which means the OCP problem can be considered as two sub-OCPs, say OCP1 and OCP2, and such partitioning of state variables is not always possible. The OCP1 related to $y_{0}-y_{1}$ control using $u_{0}-u_{1}$ and OCP1 can be solved completely independent from OCP2. Since there are directed edges from $S_{1}$ to $S_{2}$, OCP2 depends on OCP1. The subsystem which corresponds to OCP1 is a linear time invariant system with has two zero eigenvalues. On the other hand, OCP2 deals with a sluggish system of ODEs as compared to OCP1.

\section{B. State Estimation and Optimal Control}

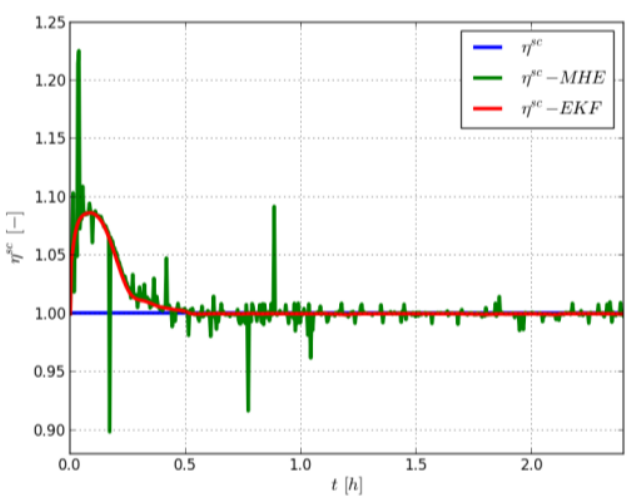

Fig. 8. Estimation of scaled $\eta$ with $N_{m h e}=2$

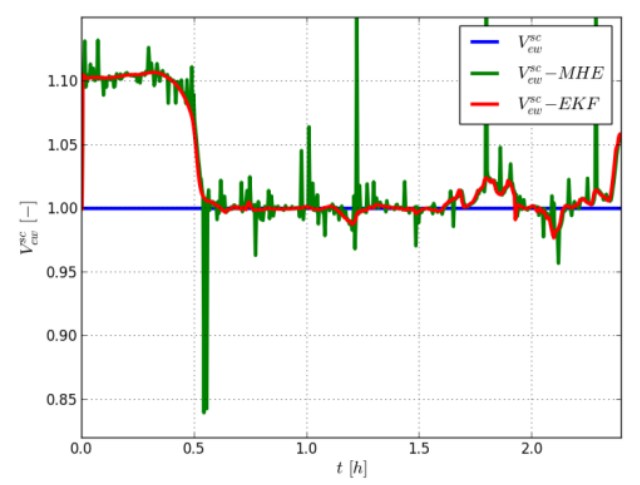

Fig. 9. Estimation of scaled $V_{e w}$ with $N_{m h e}=2$.

The model (Eq. 21) is simulated for given $w^{(1)}$ (see Fig. 22), $w^{(2)}$, and $p$ with the optimal controller given in Eq. 29. EKF 
and MHE is implemented for simulated data. Figs. 8 and 9 show estimates for $\eta$ and $V_{e w}$ using a shorter moving horizon $N_{\text {mhe }}=2$.

Note that a modified EKF with a stability correction is used here. Estimates for chemical compositions and dilution and mixing tanks' volumes are given in Figs. 11, 12, 13, 14, 15, 16, and 17 while Figs. 18 and 19 are for the estimation of disturbance variables. The controller makes outputs to follow the reference trajectories in the presence of both known and unknown disturbances - see Figs. 20 and 21.

In general, initial state estimate is unknown. However, we know that all state variables must be positive. It is important to check the stability characteristic of the estimates with respect to initial state estimate. A Monte Carlo simulation is done by choosing initial state randomly by means of uniform probability distributions. It is observed from Monte Carlo simulations - by picking initial state estimates within $\pm 50 \%$ of their nominal values - , the estimates always converging for true values. See Figs. 24 and 25. Similar Monte Carlo simulation is done to check the effect of $\alpha$. See Figs. 26 and 27. In Fig. 27, all curves converge to true value except one which corresponds to $\alpha=0$. This is an important observation. The conventional EKF fails (i.e. $\alpha=0$ ) even though the augmented state is observable. A properly tuned Moving Horizon Estimate gives comparatively better stable estimates. One of the disadvantage with the Moving Horizon Estimate is that it demand more computer resources. For $N_{m h e}=2$, the time taken per each sample time is around 1 [min] while 20 [min] for $N_{m h e}=20$. See Figs. 30 and 31 for the results when $N_{m h e}=20$.

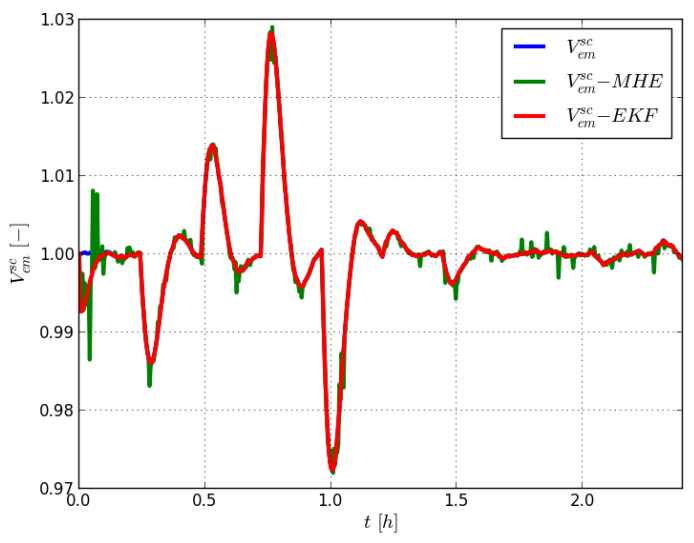

Fig. 10. Estimation of scaled $V_{e m}$ with $N_{m h e}=2$.

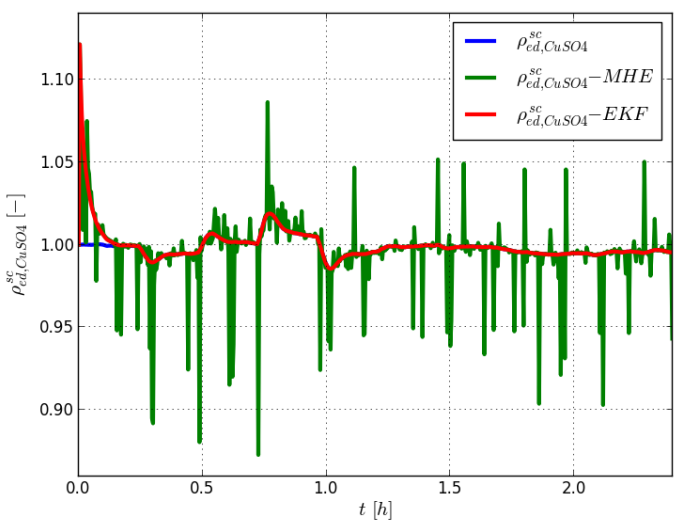

Fig. 11. Estimation of scaled $\rho_{e d, \mathrm{CuSO} 4}$ with $N_{m h e}=2$.

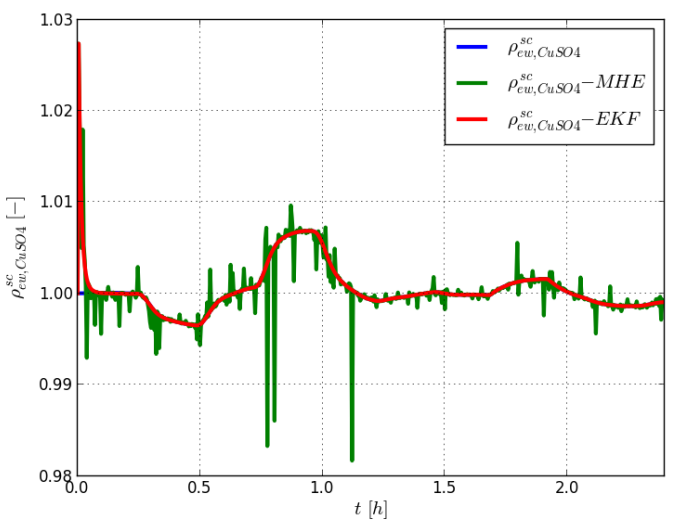

Fig. 12. Estimation of scaled $\rho_{e w, C u S O 4}$ with $N_{m h e}=2$.



Fig. 13. Estimation of scaled $\rho_{e m, C u S O 4}$ with $N_{m h e}=2$

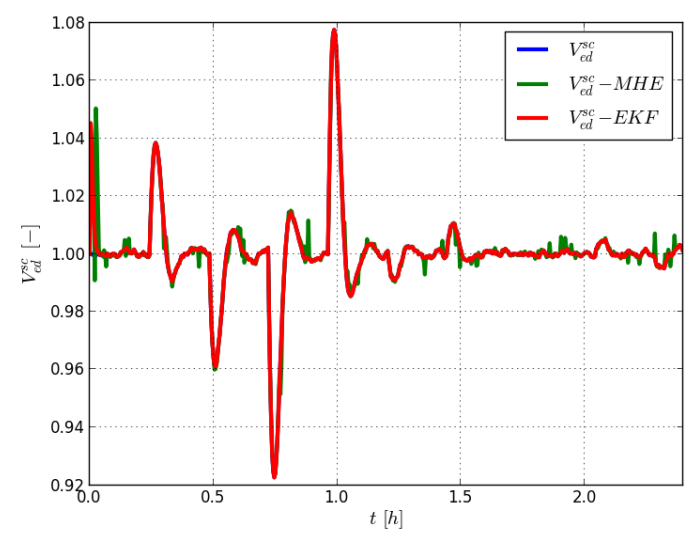

Fig. 14. Estimation of scaled $V_{e d}$ with $N_{m h e}=2$.

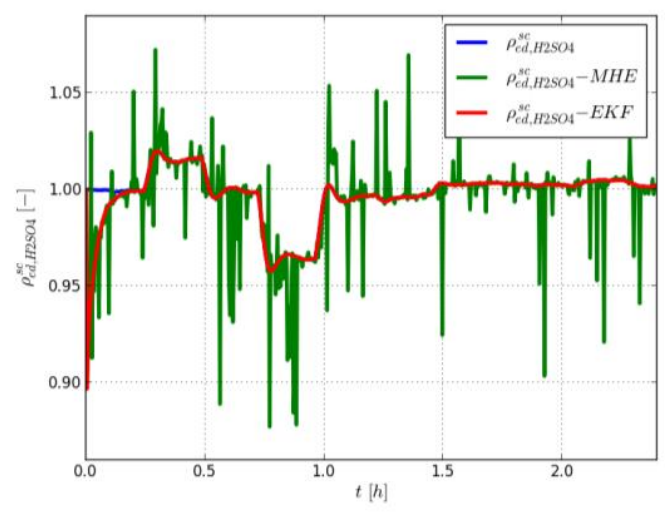

Fig. 15. Estimation of scaled $\rho_{e d, H 2 S O 4}$ with $N_{m h e}=2$ 


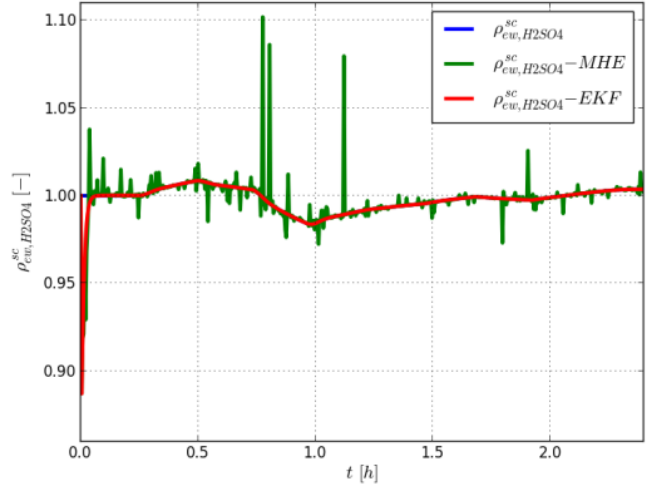

Fig. 16. Estimation of scaled $\rho_{e w, H 2 S O 4}$ with $N_{m h e}=2$.

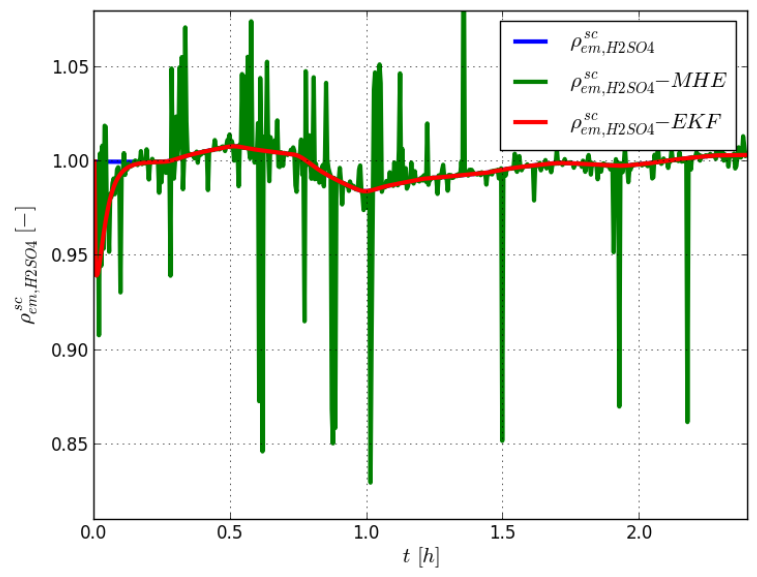

Fig. 17. Estimation of scaled $\rho_{e m, H 2 S O 4}$ with $N_{m h e}=2$.

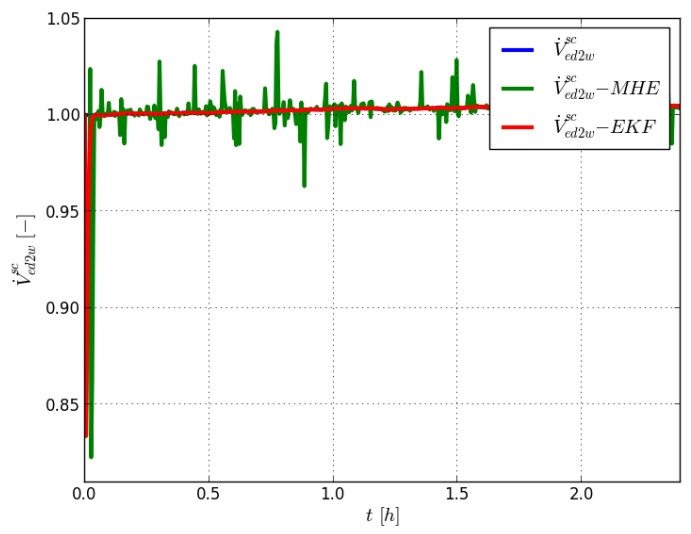

Fig. 18. Estimation of scaled $\dot{V}_{e d 2 w}$ with $N_{m h e}=2$.

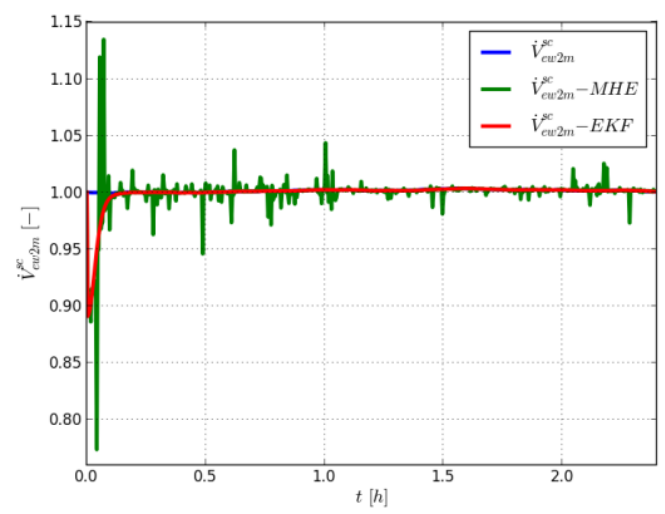

Fig. 19. Estimation of scaled $\dot{V}_{e w 2 m}$ with $N_{m h e}=2$.

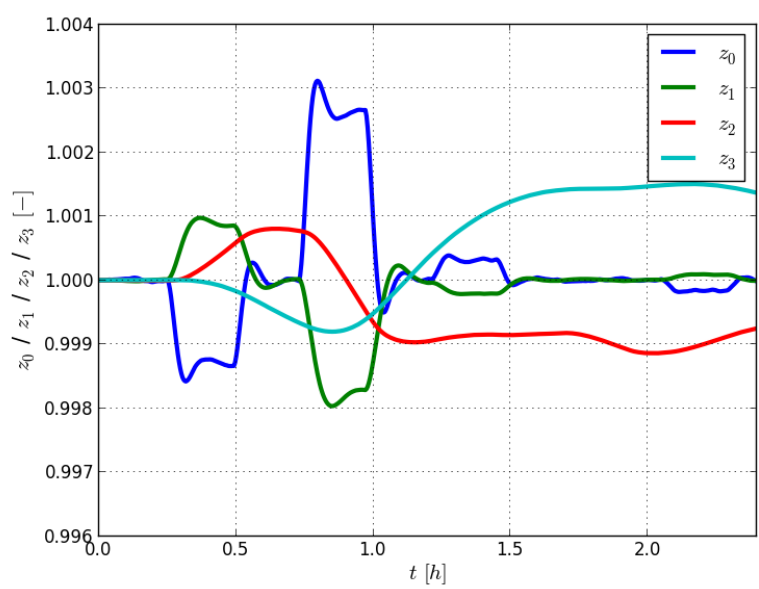

Fig. 20. Inputs.

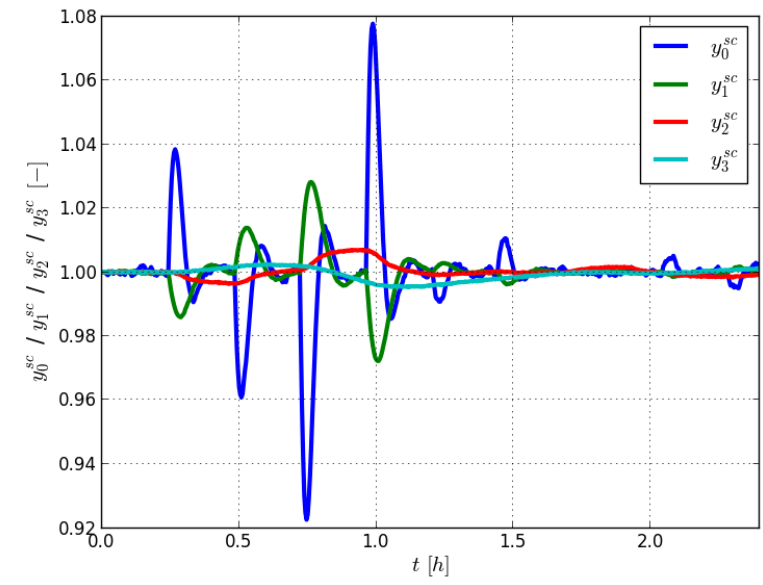

Fig. 21. Outputs.

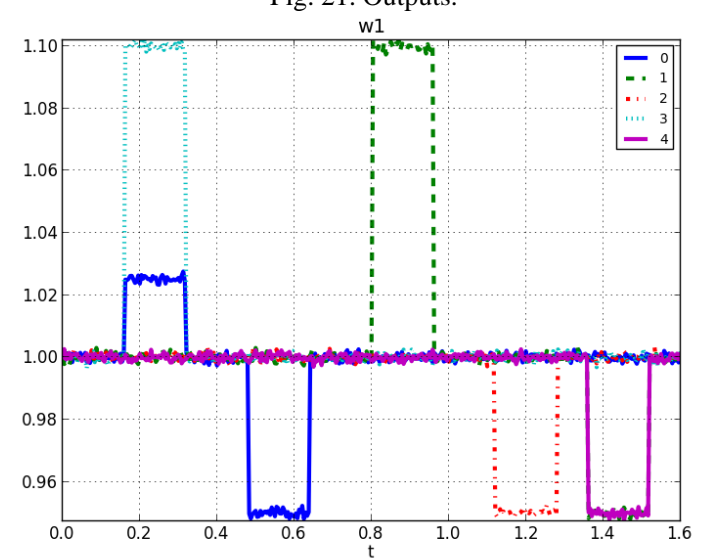

Fig. 22. Known disturbances.

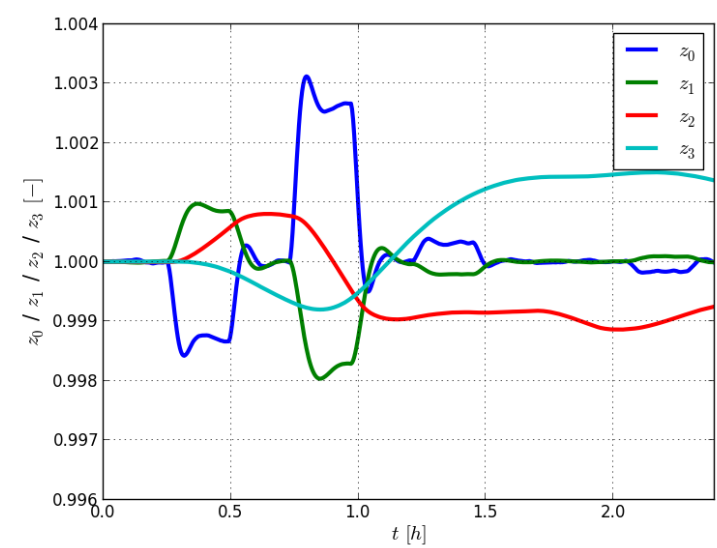

Fig. 23. Integral actions. 


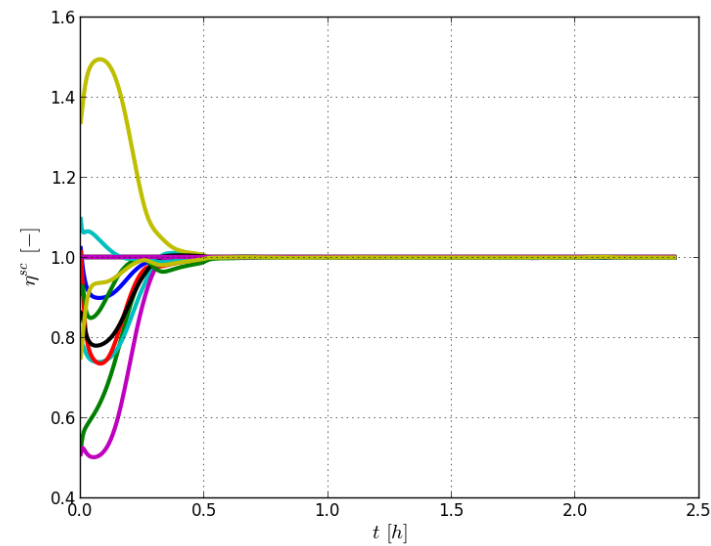

Fig. 24. Sensitivity of initial state estimates for scaled $\eta$ estimation using EKF.

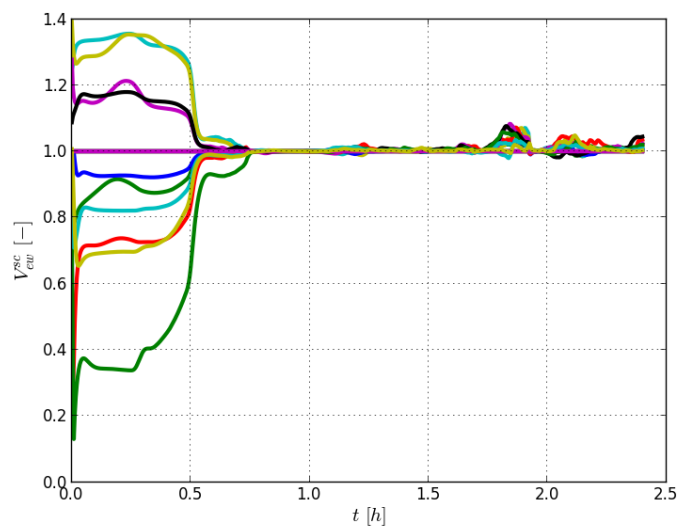

Fig. 25. Sensitivity of initial state estimates for scaled $V_{e w}$ estimation using EKF.

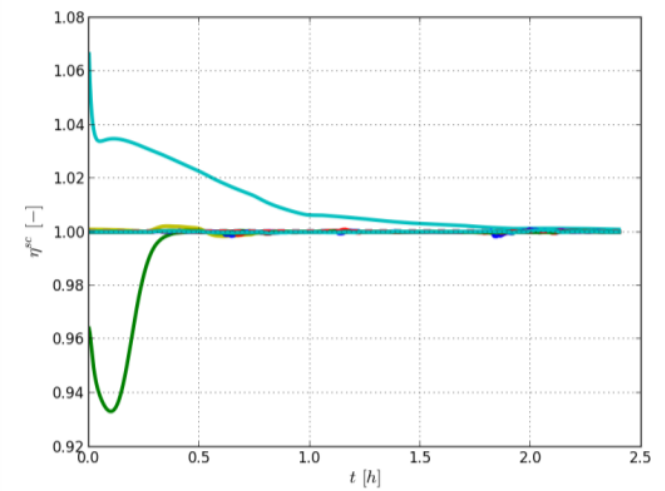

Fig. 26. Sensitivity of $\alpha$ for scaled $\eta$ estimation using EKF.

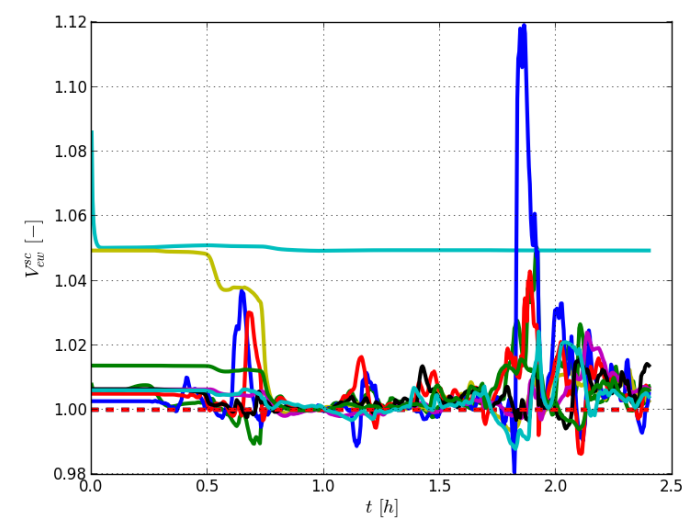

Fig. 27. Sensitivity of $\alpha$ for scaled $V_{e w}$ estimation using EKF.

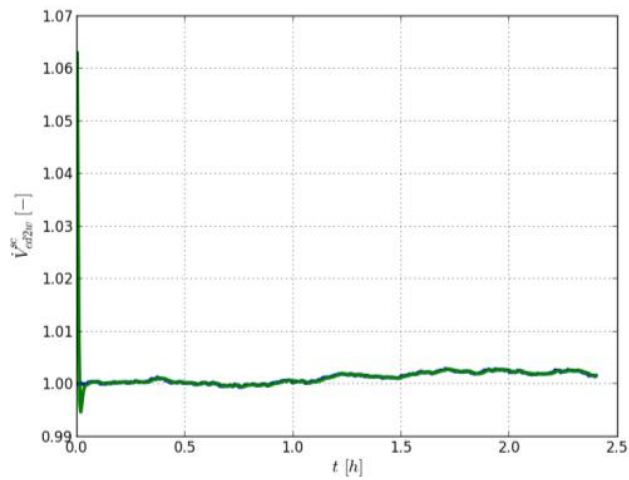

Fig. 28. Sensitivity of $\beta$ for scaled $\dot{V}_{e d 2 w}$ estimation using EKF.

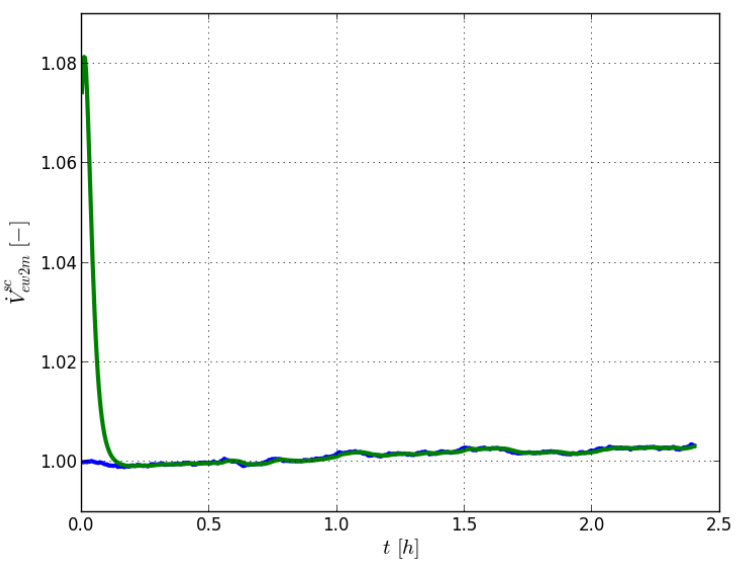

Fig. 29. Sensitivity of $\beta$ for scaled $V_{e w 2 m}$ estimation using EKF.

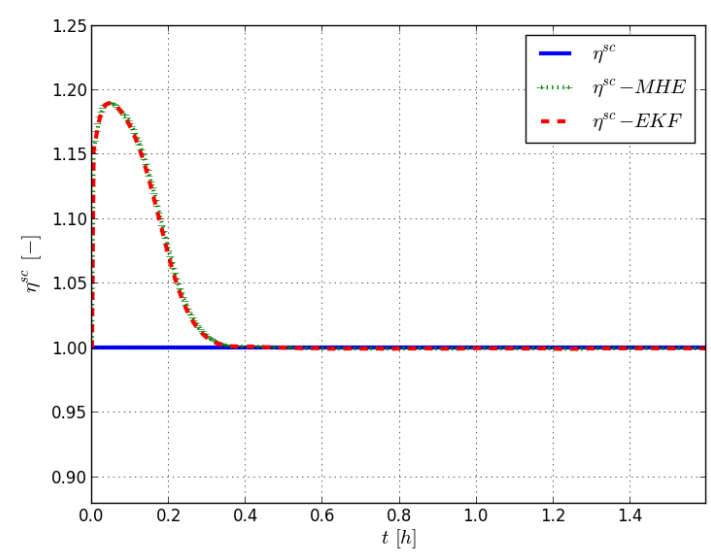

Fig. 30. Estimation of $\eta$ with $N_{m h e}=20$.

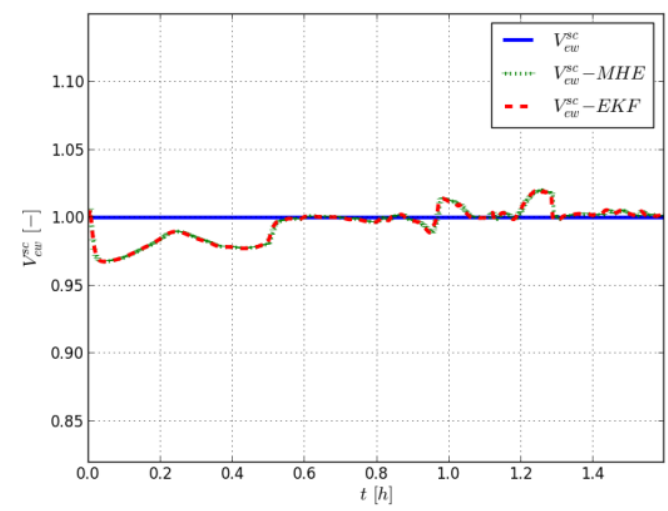

Fig. 31. Estimation of $V_{e w}$ with $N_{m h e}=20$. 


\section{CONCLUSION AND FUtURE WORK}

We have demonstrated how to use available free software and analytical tools in automating control and estimation problems related to large-scale complex dynamic systems by considering the copper leaching process at Glencore Nikkelverk, Kristiansand as a case study. The case study is a success story of exploiting structural analysis, graph-theory, Modelica and Python in handling large-scale complex control systems. The case study mainly concern optimal control and state-parameter-disturbance estimation problems and these problems connect with controllability and observability analysis. By mapping system structure appropriately into directed graphs (so-called digraphs) makes easy to analyze for structural controllability/observability. We have suggested a simple way of creating system digraphs and structural analysis using Modelica and Python.

The electrowinning process is a subsystem of the copper leaching process. A mechanistic model for the electrowinning process is available and it is considered in our analysis. The model has 8 state variables, 2 unknown disturbances and 2 unknown parameters and all of them are estimated based on available 2 level and 2 composition measurements. Two state estimators are used: the Extended Kalman Filter and the Moving Horizon Estimate. The Moving Horizon Estimator can handle constraints (e.g. chemical composition is always positive) while the Extended Kalman Filter cannot. Also, the Extended Kalman Filter may give poor stability properties. We implemented a modified Extended Kalman Filter, by replacing system matrix $A$ by $A+\alpha I$ where $\alpha$ is small positive number, which gives improved results as the Moving Horizon Estimate's results. We have shown that the Moving Horizon Estimate gives better results in sense of estimator's stability and robustness proving that the estimator is tuned properly as compared to conventional Extended Kalman Filter without including stability correction.

Before implementing any state estimator in order to estimate disturbance variables, it is essential to define dynamic models for them. There could be many possibilities of doing so. Disturbance models should be defined in such way that augmented model is structurally observable. We have shown an easy and efficient procedure to pick suitable disturbance models using a graph-theocratic approach. On the other hand, to solve the control problem, an optimal controller with integral action is implemented. The model used in controller analysis and synthesis is an augmented model and its state variables consists of both state variables in the original system and unknown disturbance variables. Here, disturbance models are defined such that unstable modes of the augmented system is controllable with respect to input variables and again, we have used the help of structural controllability analysis.

In the software implementation, we only concerned free software tools. Modeling is done in Modelica and Modelica models are used in structural observability and controllability analysis by importing Modelica models into Python via the JModelica.org-CasADi interface. Python packages NetworkX and PyGraphviz are used for graph-theocratic analysis and visualization respectively. To solve nonlinear optimization problems - which encounter in optimal control and state estimation using the Moving Horizon Estimate - constrained optimizers available in SciPy, such as fmin_cobyla, can be used. Alternatively, Ipopt (Interior Point OPTimizer) optimizer may be used and for instance, it can be accessed through CasADi.

Several suggestions are made for possible future work. An immediate extension to our work is to validate the results based on real process data. For example, state-parameter-disturbance estimation using real process data should be done. It is seen that chemical compositions in electrowinning tanks and the mixing tank are more or less equal according to model simulations, hence it is possible to consider some reduced order model in the analysis. We have considered the chemical compositions in the dilution tank as two of the control signals to control chemical compositions in the electrowinning tanks. In reality, chemical compositions in the dilution tank are manipulated by adding copper oxide and sulfuric acid at two remote locations within the copper plant and this adds imminent delays in the control action. It is necessary to include delays in the analysis. Finally, it is of interest to integrate an optimal controller to the real process and to implement an online state-parameter-disturbance estimator.

\section{REFERENCES}

[1] A. E. Bryson, Applied Optimal Control: Optimization, Estimation and Control, CRC Press, 1975.

[2] B. Lie and T. A. Hauge, "Modeling of an industrial copper leaching and electrowinning process, with validation against experimental data," in Proc. the SIMS, 2008, pp. 7-8.

[3] F. Allgöwer, R. Findeisen and C. Ebenbauer. Nonlinear model predictive control. (2000). [Online]. Available: http://ifatwww.et.uni-magdeburg.de/syst/about_us/people/findeisen/p apers/EOLSS.pdf

[4] P. Fritzson, Introduction to Modeling and Simulation of Technical and Physical Systems with Modelica, John Wiley \& Sons, 2011.

[5] J. Andersson, J. Åkesson, and M. Diehl, "Casadi: A symbolic package for automatic differentiation and optimal control," Recent Advances in Algorithmic Differentiation, Springer, 2012, pp. 297-307.

[6] K. J. Reinschke, Multivariable Control: A Graph Theoretic Approach, Springer-Verlag, 1988.

[7] D. P. Bertsekas, "Dynamic programming and optimal control," Athena Scientific Belmont, Massachusetts, vol. 1, 1996.

[8] L. T. Biegler, Nonlinear Programming: Concepts, Algorithms, and Applications to Chemical Processes, vol. 10, 2010.

[9] L. Magni, D. M. Raimondo, and F. Allgöwer, Nonlinear Model Predictive Control, Springer, 2009.

[10] D. Simon, Optimal State Estimation: Kalman, $H$ Infinity, and Nonlinear Approaches, Wiley-Interscience, 2006.

[11] R. Brown and P. Hwang, Introduction to Random Signals and Applied Kalman Filtering - With MATLAB Exercises and Solutions, John Wiley and Sons, 1997.

[12] A. Gelb, Applied Optimal Estimation, The M.I.T. press, 2001.

[13] A. H. Jazwinski, Stochastic Processes and Filtering Theory, Courier Corporation, 2007.

[14] A. Jazwinski, "Limited memory optimal filtering," IEEE Transactions on Automatic Control, vol. 13, no. 5, 1968, pp. 558-563.

[15] K. Reif, F. Sonnemann, and R. Unbehauen, "Modification of the extended kalman filter with an additive term of instability," in Proc. the 35th IEEE Conference on Decision and Control, vol. 4, 1996, pp. 4058-4059.

[16] R. J. Fitzgerald, "Divergence of the kalman filter, automatic control," vol. 16 , no. 6, 1971, pp. 736-747.

[17] L. Ljung, "Asymptotic behavior of the extended kalman filter as a parameter estimator for linear systems, automatic control," vol. 24, no. 1, 1979, pp. 36-50.

[18] B. Bona and R. J. Smay, "Optimum reset of ship's inertial navigation system, aerospace and electronic systems," IEEE Transactions on $A E S$-2, vol. 4, 1966, pp. 409-414.

[19] A. Doucet, S. Godsill, and C. Andrieu, "On sequential monte carlo sampling methods for bayesian filtering," Statistics and Computing, vol. 10 , no. 3, 2000, pp. 197-208. 
[20] N. Gordon, B. Ristic, and S. Arulampalam, Beyond the Kalman Filter: Particle Filters for Tracking Applications, Artech House, London.

[21] A. Smith, A. Doucet, N. de Freitas, and N. Gordon, Sequential Monte Carlo Methods in Practice, Springer Science \& Business Media, 2013.

[22] D. F. Bizup and D. E. Brown, "The over-extended kalman filter-don't use it!," in Proc. the Sixth International Conference of Information Fusion, vol. 1, 2003, pp. 40-46.

[23] K. J. Åström, Introduction to Control, Department of Automatic Control, Lund Institute of Technology, 2004

[24] P. C. Young and J. Willems, "An approach to the linear multivariable servomechanism problemâ€," International Journal of Control, vol. 15 no. 5, 1972, pp. 961-979.

[25] Y.-P. Shih, "Integral action in the optimal control of linear systems with quadratic performance index," Industrial and Engineering Chemistry Fundamentals, vol. 9, no. 1, 1970, pp. 35-37.

[26] A. Isidori, Nonlinear Control Systems, Springer Science \& Business Media, 1995.

[27] R. Hermann and A. J. Krener, "Nonlinear controllability and observability," IEEE Transactions on Automatic Control, vol. 22, no. 5, 1977, pp. 728-740.

[28] C. T. Lin, Structural Controllability, Automatic Control, vol. 19, no. 3, 1974, pp. 201-208.

[29] Y.-Y. Liu, J.-J. Slotine, and A.-L. Barabási, "Observability of complex systems," in Proc. the National Academy of Sciences, vol. 110, no. 7 , 2013, pp. 2460-2465.

[30] M. R. James, "Controllability and observability of nonlinear systems," Tech. rep., Mathematics Department and Systems Research Center, University of Maryland, 1987.

[31] M. A. S. Perera, B. Lie, and C. F. Pfeiffer, "Structural observability analysis of large scale systems using modelica and python, modeling," Identification and Control, vol. 36, no. 1, 2015, pp. 53-65.

[32] J. E. Potter, "A matrix equation arising in statistical filter theory," Tech. rep., National Aeronautics and Space Administration, 1965.

[33] J. Åkesson, K.-E. Årzén, M. Gäfvert, T. Bergdahl, and H. Tummescheit, "Modeling and optimization with optimica and jmodelica.Org languages and tools for solving large-scale dynamic optimization problems," Computers and Chemical Engineering, vol. 34 , no. 11,2010 , pp. 1737-1749.

[34] A. Wächter and L. T. Biegler, "On the implementation of an interior-point filter line-search algorithm for large-scale nonlinear programming," Mathematical Programming, vol. 106, no. 1, 2006, pp. $25-57$.

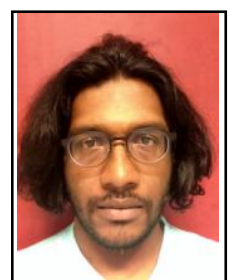

M. Anushka S. Perera was born in 1982 in Negombo, Sri Lanka. He received his bachelor's degree in chemical and process engineering from University of Moratuwa, Sri Lanka in 2007 and master's degree in systems and control engineering from Telemark University College, Porsgrunn, Norway (at time it was called Telemark University College) in 2012. He is currently a PhD candidate at the University College of Southeast Norway, Porsgrunn. His main research complex control systems. interests are estimation and control of large-scale

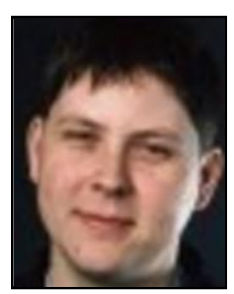

Tor Anders Hauge obtained his $\mathrm{PhD}$ from Telemark University College, Porsgrunn, Norway in 2003. He has worked as adjunct professor at University of Agder's mechatronics group during the course of 2008-2011. He has been working as a control engineer from 2003 at Glencore Nikkelverk, Kristiansand, Norway and his current position is senior control engineer.

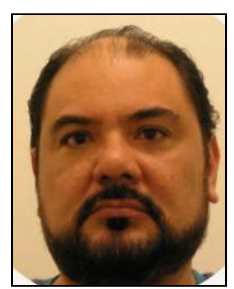

Carlos Fernando Pfeiffer obtained his bachelor's degree in chemical and systems engineering from ITESM, Monterrey, Mexico in 1987, a master's degree in control engineering degree from the same institution in 1993 and the $\mathrm{PhD}$ from the University of Texas at Austin in 1999. The topic of his dissertation was heterogeneous control laws for nonlinear systems. He worked as a process engineering at the Advanced Process Research and theDevelopment Center at Motorola, Austin, from 1998 to 2001, implementing advanced control and monitoring for the lithography process, etch process and chemical mechanical planarization (CMP). Carlos F. Pfeiffer was a professor at the Computer Science Department at ITESM, Monterry, Mexico from 2001 to 2010 . From 2011 to date, he is faculty at Telemark University College, now University College of Southeast Norway. His current fields of teaching and research are advanced process control, process modeling and optimization, thermodynamics, computer vision, pattern recognition. Present research projects: human behavior modeling for smart house and welfare technology, thermodynamic characterization of $\mathrm{CO} 2$ in polymer solutions, nonlinear process control. 